\title{
GNSS ANTENNA INTEGRATED INTO A CLASSICAL FIBRE METAL LAMINATE FUSELAGE PANEL
}

\author{
EMuS 2020 \\ Y. KONTER*, C. HEUTS ${ }^{\dagger}$, C. VAN HENGEL ${ }^{\ddagger}$, J. VERPOORTE $^{*}$ \\ *Royal Netherlands Aerospace Centre (NLR) \\ Voorsterweg 31, 8316 PR Marknesse, Netherlands \\ e-mail: Yuri.Konter@nlr.nl, web page: https://www.nlr.nl \\ $\dagger$ GKN Fokker Elmo \\ Aviolandalaan 33, $4631 \mathrm{RP}$, Netherlands \\ e-mail: Charly.Heuts@fokker.com, web page: http://www.gknaerospace.com \\ $¥$ GKN Fokker Aerostructures \\ Industrieweg 4, 3351 LB Papendrecht, Netherlands \\ e-mail: Cees.vanHengel@fokker.com, web page: http://www.gknaerospace.com
}

Key words: Integrated Antenna, GLARE, GNSS double patch antenna

\begin{abstract}
Fibre Metal Laminate materials, like Glass Laminate Aluminum Reinforced Epoxy (GLARE), provide a unique opportunity to integrate patch antennas directly into the fuselage of aircraft. This integration will contribute to the reduction of carbon emissions of aircraft due to the reduction of aerodynamic drag. This paper discusses the integration of a double patch GNSS antenna directly into a classical Fiber Metal Laminate. A demonstrator of the antenna was manufactured and measured. The antenna has a flush finish on the outside of the fuselage. The preliminary results of these measurements show promising antenna performance, but more measurements are required to fully characterize this integrated antenna.
\end{abstract}

\section{INTRODUCTION}

The goal of the European Union Horizon2020 ACASIAS project is to reduce the environmental impact of aircraft by improving aerodynamics and reduction of the weight of the aircraft. The aerodynamic drag and the weight of the aircraft are being reduced by integrating antennas and sensors in the structure of the aircraft. Conventional antennas are installed as protruding elements on the exterior of fuselages. These protruding elements increase the aerodynamic drag of an aircraft. Fibre Metal Laminate materials (FML) provide an opportunity to realize integrated antenna designs that can eliminate the requirement for protruding antennas.

This paper will address the integration of a Global Navigation Satellite System (GNSS) antenna in the FML skin of the aircraft. GNSS radiation in the L1 and L5 bands is realized using two concentric circular radiating patches. Good axial ratio is achieved by feeding the patches with four probes [1]. The four probes are combined on the inside of the fuselage using a hybrid coupler printed circuit board. The resulting integrated antenna element is flush on the outside of the fuselage. 
A demonstrator of the FML panel with integrated GNSS antenna has been made and measured. These measures will be discussed. Simulation and measurement results of the integrated GNSS antenna will be discussed.

\section{DESIGN OF THE DOUBLE BAND INTEGRATED GNSS ANTENNA}

The integrated GNSS antenna is created by modifying a standard GLARE panel in order to accommodate the electrical elements required by the antenna. The Fibre Metal Laminate (FML) GLARE is build-up of $0.3 \mathrm{~mm}$ thick aluminium and $0.13 \mathrm{~mm}$ thick glass-fibre layers. The integrated GNSS antenna is designed using the same GLARE materials. This allows the antenna patches to align with the GLARE aluminium panels.

The antenna is designed as a stacked patch antenna using circular conducting patches as radiating elements. Two patches are used which are tuned to radiate in the L1 and L5 GNSS bands. The patches are stacked concentrically as shown in Figure 1 A. The L5 patch is made of aluminium, similar to remaining GLARE panel. The smaller L1 patch is made of copper in order to allow galvanic connection of feeds to the patch.
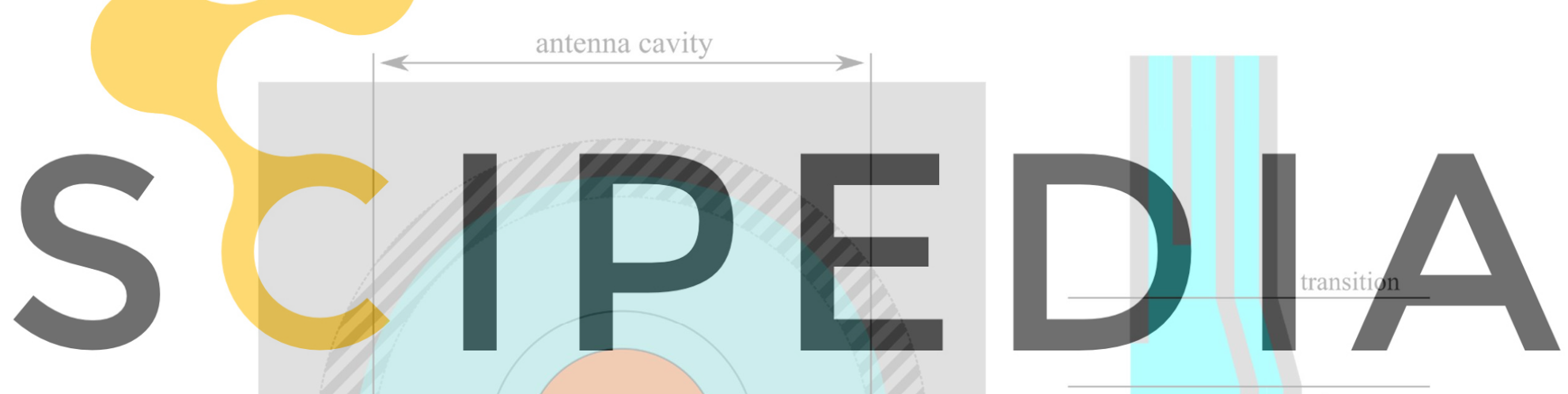

Register for free at https//www.scipedia.com to download
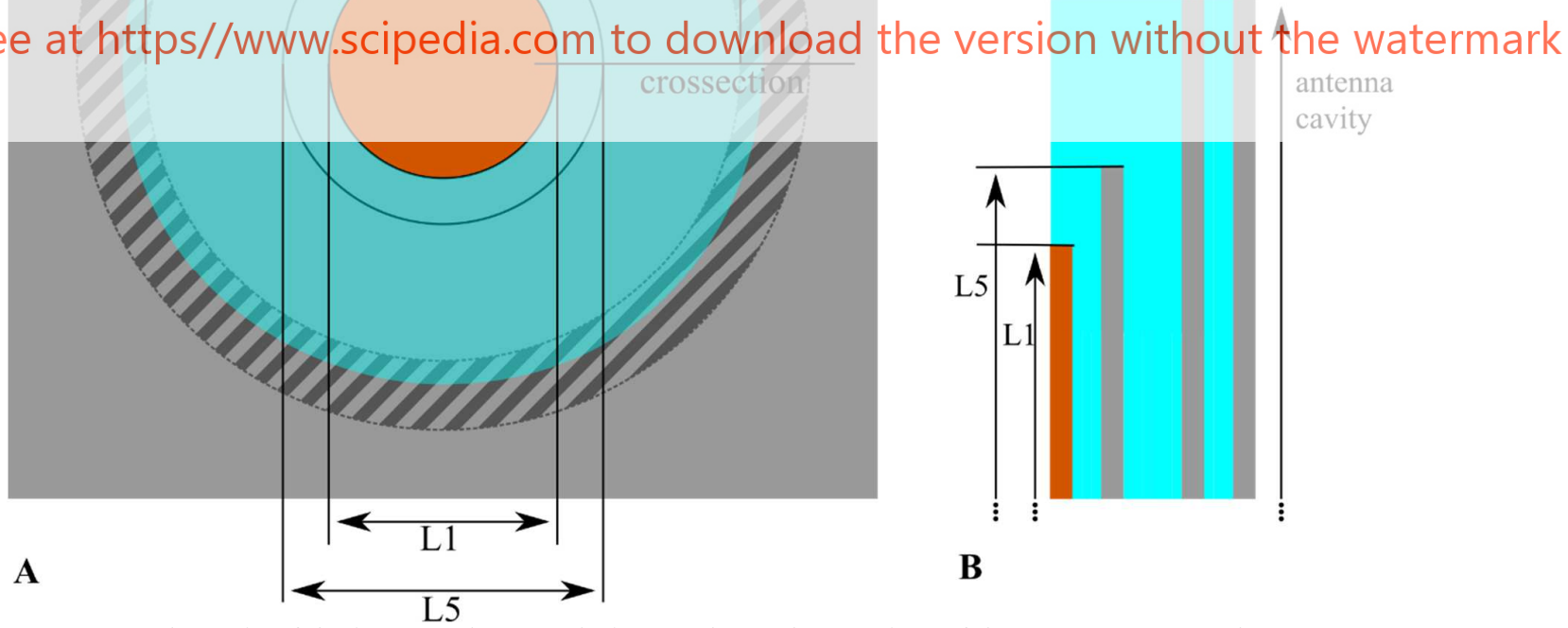

Figure 1: Schematic of the integrated GNSS design. A shows the top view of the GNSS antenna. The two circular antenna patches L1 (orange) and L5 (grey) and the resin window (blue). The transition from the antenna cavity into regular GLARE is shown by the hatched area. B shows a cros-section of the FML panel, indicating how the layers of material are stacked to create the cavity for the GNSS antenna. 
The antenna ground-plane is realized as a circular dent in the inner aluminium layer. A transition area is introduced (Figure $1 \mathrm{~B}$ ) in order to fully integrate the antenna in a regular GLARE panel.

\subsection{Antenna Feed}

The L1 patch of the dual band antenna is fed with four probes. This feed method, combined with the circular radiating patches, has been shown to give good axial ratio performance for a wide frequency-band [1]. The location of the feeds is shown in Figure 2 B. The L5 patch is capacitively coupled to the L1 patch, therefore a hole is drilled to avoid a galvanic connection between the feed probes and the L5 circular patch. The feeds are positioned such that the input impedance of the feeds is closest to $50 \Omega$ for both the L1 and the L 5 frequency bands.

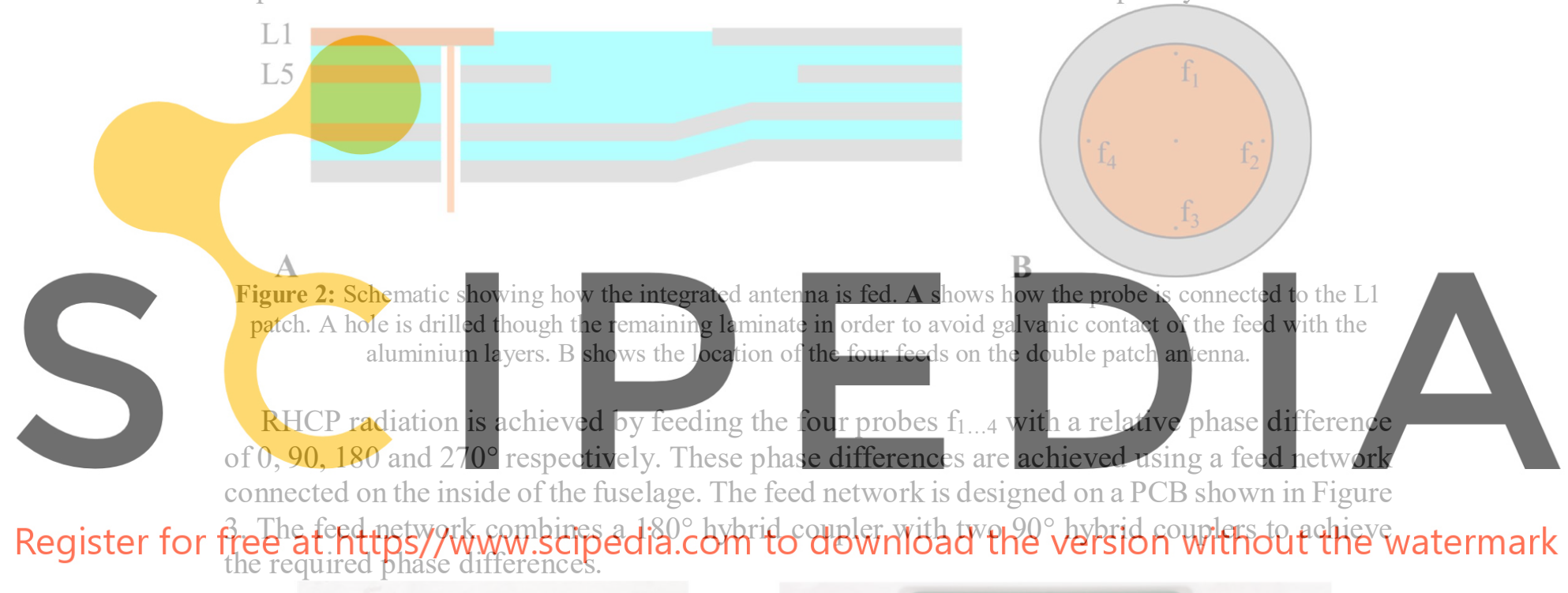

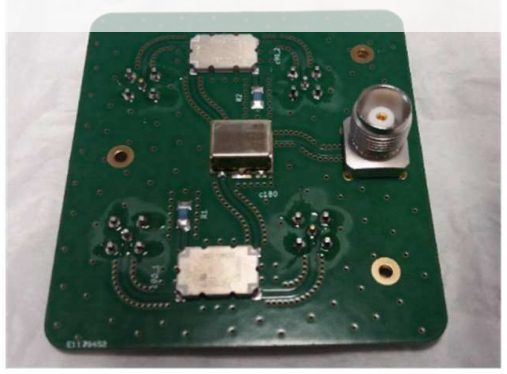

A

Figure 3: PCB with feed network to achieve phase difference for the four probes. A shows the three surface mount devices used to achieve the phase differences. It also shows the single output connector for the antenna signal. B shows the four connectors used to attach the PCB to the inside of the fuselage.

Finally, a grounded via is added in the centre of circles to prevent the build-up of static charge on the two antenna patches. This via is galvanically connected to both the L1 and the L5 patches. 


\section{RF PERFORMANCE OF THE GNSS ANTENNA DEMONSTRATOR}

A demonstrator of the integrated antenna was manufactured in a 400 by $400 \mathrm{~mm}$ GLARE panel. The antenna patches where placed in the autoclave together with the materials for the GLARE panel. The resulting integrated antenna is shown in Figure 4.

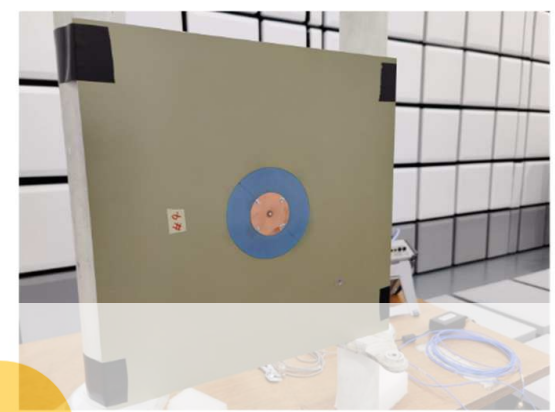

A

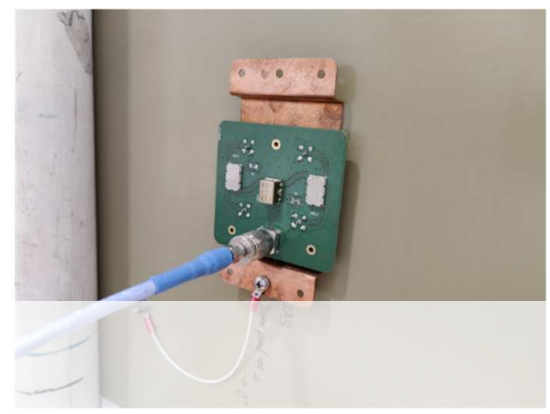

B

Figure 4: Two images of the integrated GNSS antenna demonstrator. A shows the antenna as seen from the outside of the fuselage. B shows the antenna feed network connected to the integrated antenna on the inside of the fuselage.

The antenna is fully integrated in the GLARE panel and has a flush finish on the outside of fuselage. The copper patch used for the L1 radiation is visible from the outside of the fuselage,

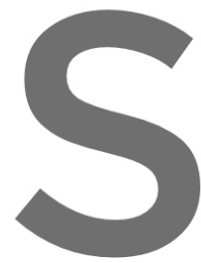
together with the glas fuselage as shown in fuselage.

\section{Register for free at-shttps//www.scipedia.com to download the version without the watermark}

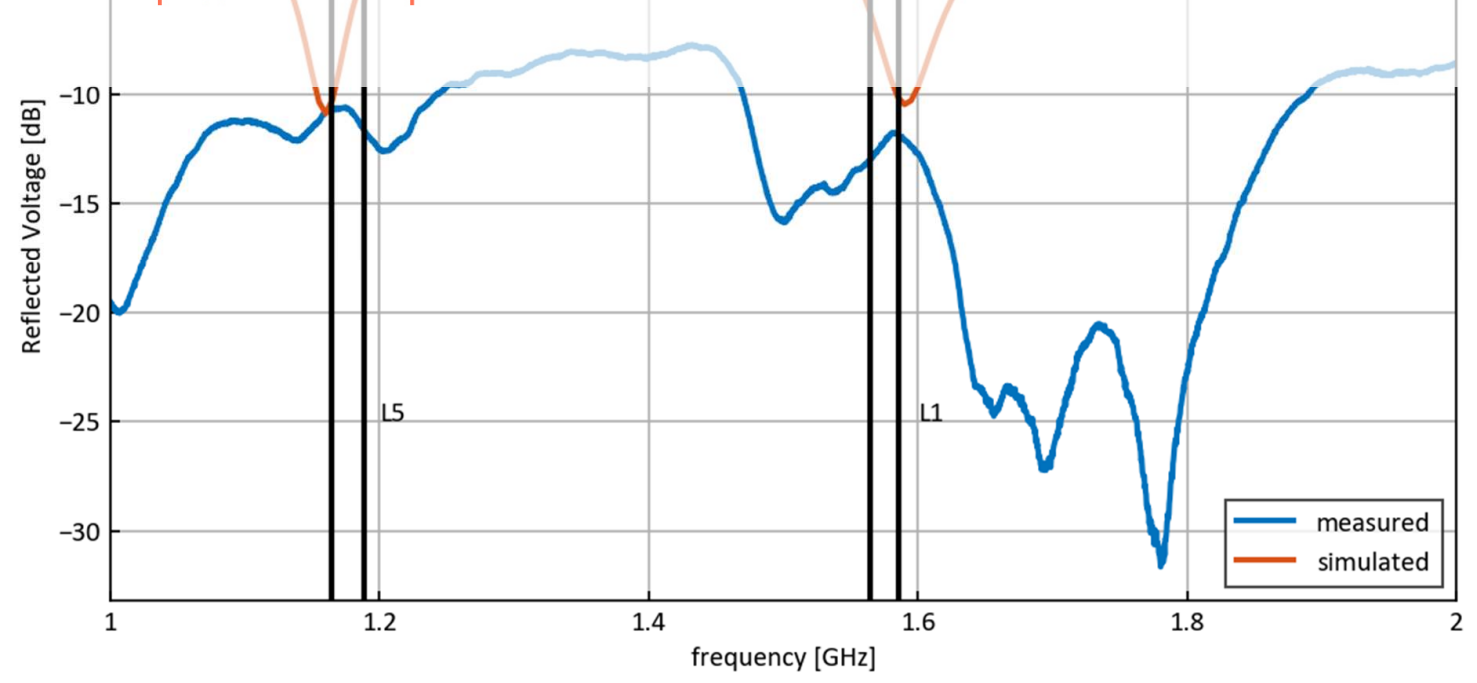

Figure 5: Simulated and measured reflected voltage of the antenna demonstrator. The L1 and L5 GNSS frequency bands are indicated by the black vertical lines. 


\subsection{Reflected voltage}

The reflected voltage of the antenna demonstrator was measured using the vector network analyse. The measurement results are shown in Figure 5. The measured reflected voltage is lower than the required $-10 \mathrm{~dB}$ target within the L1 and L5 GNSS frequency bands. This indicate that the antenna will work within these frequency bands.

Notably, the measured reflected voltage does not correspond well with the simulations performed in HFSS. The similarity between results can likely be improved if the reflected voltage of the demonstrator antenna is measured without the feed network. This was not possible at the time of writing due to missing parts required to perform this measurement.

Moreover, shifts in resonance frequency are likely caused by uncertainty of the electromagnetic properties of the glass fibre layers used in the GLARE panel or by fabrication inconsistencies of the demonstrator panel. More measurements are required to identify the nature of the frequency shifts.

\subsection{Radiation pattern}

The radiation pattern of the antenna was also measured for the L1 GNSS frequency band. The measurement setup is shown in Figure 6. The setup was used to measure the angular dependence of the antenna gain. These gains are shown in Figure 7.
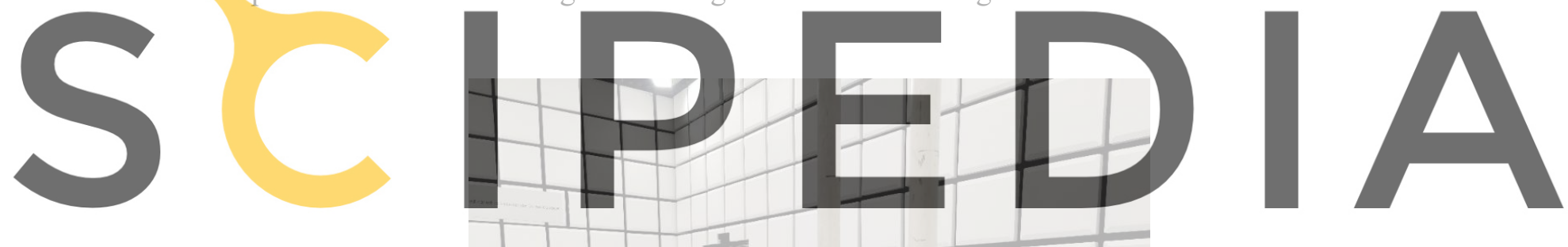

Register for free at https//www.scipedia.com to download the version without the watermark



Figure 6: Measuring setup used in measuring the radiation pattern of the antenna demonstrator. A Right Handed Circular Polarized (RHCP) antenna or a Left Handed Circular Polarized (LHCP) antenna is placed on the far side of the room. The antenna demonstrator is rotated from -90 to 90 degrees. 
frequency $=1575 \mathrm{MHz}$

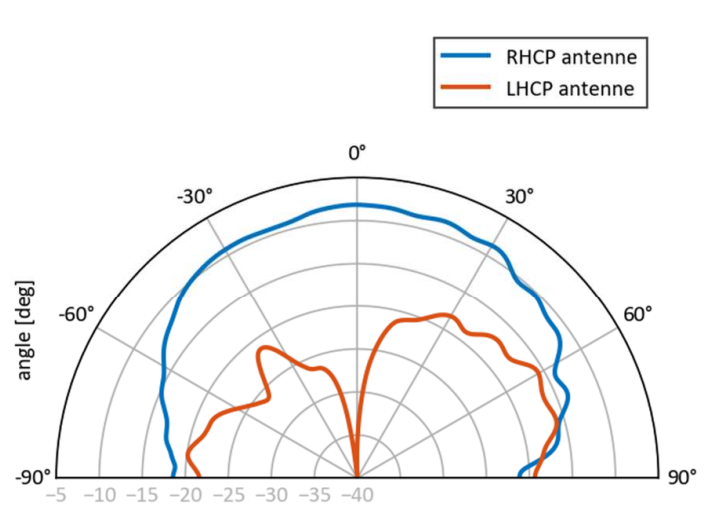

gain [dBic] frequency $=1610 \mathrm{MHz}$

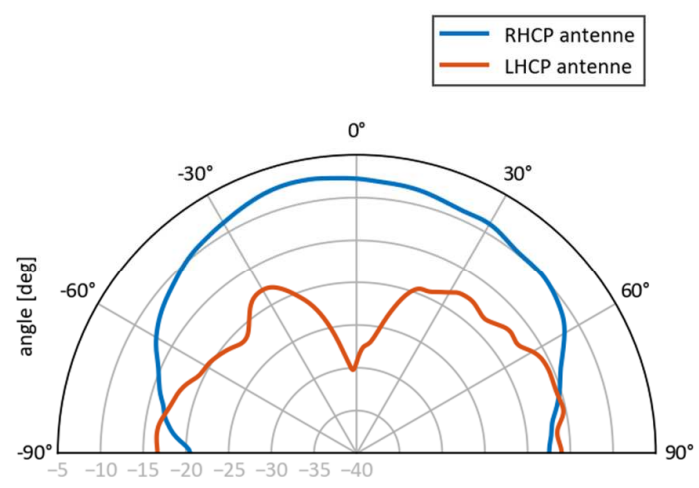

gain $[\mathrm{dBic}]$

Figure 7: Measured RHCP and LHCP gain of the integrated GNSS antenna demonstrator at 1575 and 1610 $\mathrm{MHz}$.

The measurements show good RHCP gain for a broad viewing angle. However, the total gain of the antenna is lower than expected. This could be either due to additional losses in the GLARE materials, or due to issues with the feed network. More measurements are required to pinpoint the exact cause of these losses. Nevertheless, the (LHCP) radiation graphs show good rejection of cross polar radiation. This indicates good polarization purity of the antenna demonstrator.

4 CONCLUSIONS
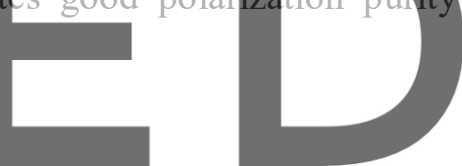

A GNSS antenna was manufactured directly into a classical GLARE FML panel. The

Register for fresulting hutens the inside of the fuselage panel. A feed network, required for RHCP radiation, was designed on

a PCB and mounted on the inside of the fuselage panel. Measurements of the antenna with the feed network show lower than $-10 \mathrm{~dB}$ reflected voltage for a broad frequency range. However, additional measurements are required to fully characterize the antenna.

The radiation pattern of the L1 GNSS frequency band was also measured. The RHCP and LHCP radiation patterns indicate good radiation purity in the L1 band. However, the gain of the antenna is lower than expected. More measurements are required to identify the cause of these losses.

\section{ACKNOWLEDGEMENTS}

This project has received funding from the European Union's Horizon2020 research and innovation programme under grant agreement No.723167.

\section{REFERENCES}

[1] K. A. Yinusa, "A Dual-Band Conformal Antenna for GNSS Applications in Small Cylindrical Structures," IEEE Antennas Wirel. Propag. Lett., vol. 17, no. 6, pp. 1056-1059, Jun. 2018, doi: 10.1109/LAWP.2018.2830969. 